\section{$\mathrm{CO}_{2}$-Bilanz und Vegetationsänderungen}

Zentralthema vieler Konferenzen der letzten Jahre war die Frage nach dem Woher und Wohin des Kohlenstoffs auf unserer Erde. Das Interesse wurde ausgelöst durch den beobachteten Anstieg an $\mathrm{CO}_{2}$ in unserer Atmosphäre und seinen möglichen Einfluß auf das Klima der nächsten 100 Jahre [1-3]. Fazit der ersten Konferenzen: Die Antwort auf die $\mathrm{CO}_{2}$-Frage liegt nicht nur in der Luft; es bedarf statt dessen ergänzend einer gründlichen Studie des globalen Kohlenstoff-Zyklus auf dem Lande, dem Wasser und der Lebewelt. Dementsprechend erweiterte sich die Diskussion in den Folgekonferenzen und damit auch die Vielfalt der Meinungen.

Sicher ist zur Zeit nur eins: Der Anstieg an $\mathrm{CO}_{2}$ in der Atmosphäre ist verursacht durch den Menschen. Es ist hier vor allen Dingen die Verbrennung von Kohle, Öl, Gas und Holz. Bis Ende dieses Jahrhunderts wird der $\mathrm{CO}_{2}$-Spiegel um etwa 15 bis $20 \%$ höher liegen als heute. Von da $a b$ werden die Voraussagen unsicher, einfach weil die $\mathrm{CO}_{2}$-Wachstumskurve nicht linear, sondern exponentiell verläuft und wir nach dem Jahre 2000 in einen Bereich kommen, wo die Kurve nicht mehr so flach ist wie heute, sondern wo sie steil nach oben geht. Wie steil, das ist der Kernpunkt, wo sich die Geister scheiden. Die Vorstellungen reichen von doppelt so hoch bis siebenmal so hoch um die Mitte des nächsten Jahrhunderts [4].

$\mathrm{CO}_{2}$ wirkt wie ein unsichtbares Treibhausdach und führt zu einem Temperaturanstieg in der Atmosphäre. Als grobe Faustregel kann man sagen, daß eine Verdoppelung des $\mathrm{CO}_{2}$-Spiegels die globale Temperatur um rund $2^{\circ} \mathrm{C}$ erhöht [4]. Aus der geologischen Vergangenheit wissen wir, daß ein Anstieg um $2^{\circ} \mathrm{C}$ zu einer durchgreifenden Verlagerung der Klimagürtel unserer Erde führen würde, mit Konsequenzen, die für unsere Gesellschaft mehr als bedrohlich sind, da die Veränderungen sich im Verlauf nur weniger Jahrzehnte vollziehen. Selbst eine, ,konservative “ Vorausberechnung - eine $\mathrm{CO}_{2}$-Verdoppelung um das Jahr 2050 - läßt die öffentliche
Meinung bislang ungerührt. Wir müssen die anthropogene Zufuhr an $\mathrm{CO}_{2}$ in die Atmosphäre drosseln, was einfacher gesagt ist, als getan.

Zur Zeit gehen jährlich rund 5 Milliarden Tonnen Kohlenstoff in Form von $\mathrm{CO}_{2}$ durch Verbrennung von Erdöl, Kohle und Erdgas in die Luft. Zusätzlich werden nicht unerhebliche $\mathrm{CO}_{2}$-Mengen ,dank" Abholzungen, vorallen Dingen in tropischen Wäldern, durch die Oxidation von Holz und Humus frei. Wie hoch dieser Betrag ist, darüber wird gestritten. Eine Forschergruppe spricht von 0,4 bis 0,8 Milliarden Tonnen Kohlenstoff [5], eine andere [6] von Werten zwischen 4 und 8 Milliarden Tonnen Kohlenstoff pro Jahr. Eine ausführliche Diskussion aller Argumente und Daten findet sich in [4]. Die Gewichtung aller Befunde spricht mehr für die höheren Werte, was bedeutet, daß jährlich rund 5 Milliarden Tonnen Kohlenstoff durch Abholzung und Humusoxidation als $\mathrm{CO}_{2}$ in die Luft entweichen.

Wir halten fest: Rund 10 Milliarden Tonnen Kohlenstoff werden durch den Menschen in Form von $\mathrm{CO}_{2}$ pro Jahr in die Atmosphäre abgegeben, und zwar zu gleichen Teilen durch Verbrennung fossiler Energieträger und Waldrodungen. Davon verbleiben zur Zeit etwa 2,5 Milliarden Tonnen als $\mathrm{CO}_{2}$ in der Atmosphäre. Der Rest, also 7,5 Milliarden Tonnen, wird irgendwo aufgenommen, sei es durch verstärkte Pflanzentätigkeit oder durch bio- geochemische Prozesse im Ozean. Auch über dieses Geschehen der Umsetzung des anthropogenen Kohlendioxids und sein Verschwinden besteht weitgehend Unklarheit. Wie verwirrend das Bild ist, verdeutlicht der nächste Satz: Der Biologe sieht die Senke im Ozean und der Ozeanograph in der Pflanzenwelt.

Es ist wichtig festzuhalten, daß die Erde selbst bislang $\sim 75 \% \quad\left(=7,5 \times 10^{9} \mathrm{t} \quad \mathrm{C} /\right.$ Jahr) des anthropogenen Kohlendioxids der Luft entzieht über Mechanismen, die wir zur Zeit nur in Ansätzen verstehen. Der Rest, also $\sim 25 \% \quad\left(=2,5 \times 10^{9} \mathrm{t} \mathrm{C}\right.$ / $\mathrm{Jahr}$ ), verbleibt in der Atmosphäre. Gelingt es uns, den Gesamtausstoß an $\mathrm{CO}_{2}$ unter $7,5 \times 10^{9} \mathrm{t} \mathrm{C} / \mathrm{Jahr}$ zu halten, so könnte der $\mathrm{CO}_{2}$-Spiegel in der Atmosphäre sich möglicherweise auf einen verträglichen Normalwert um 300 bis 400 ppm $\mathrm{CO}_{2}$ einpendeln. Die ,,Pufferwirkung" der Erde für $\mathrm{CO}_{2}$ scheint damit recht beachtlich $\mathrm{zu}$ sein.

E.T. Degens/Hamburg

1. Plass, G.N. : Tellus 8, 140 (1956)

2. Keeling, C.D., Bacastow, R.B., in: Energy and Climate, p. 72. Washington, D.C.: Nat. Acad. Sci. 1977

3. Beardsmore, D.J., et al.: The CSIRO (Australia) Atmospheric Carbon Dioxide Monitoring Program: The First Six Years of Data. Commonwealth Sci. Industr. Res. Organisation, Australia, 1978

4. Bolin, B., et al.: The Global Carbon Cycle. SCOPE 13. Chichester-New York-BrisbaneToronto: Wiley 1979

5. Ralston, C.W.: Science 204, 1345 (1979)

6. Woodwell, G.M, et al.: ibid. 204, 1346 (1979)

\title{
Biochemische Verhaltenskontrolle
}

Die Regulation zahlreicher aggregierender Proteine durch Phosphorylierung/Dephosphorylierung ist mittlerweile wohlbekannt. Unter hormoneller Steuerung durch cyclo-AMP oder durch $\mathrm{Ca}^{2+}$-Ionenverschiebungen werden Proteinkinasen aktiviert [1], die mittels ATP bestimmte Serinyl-Seitengruppen der Proteine phosphorylieren und sie zu aktiven Komplexen ag- gregieren oder auch desaggregieren lassen. Dieser Zustand kann durch enzymatische Abspaltung der Phosphatestergruppen rückgängig gemacht werden. Auch diese Phosphatasen stehen unter entsprechender hormoneller Kontrolle.

Ein weiterer Vorgang, durch den Proteine modifizierend aktiviert werden, wurde kürzlich in der Vitamin K-abhängigen 